\title{
Calcium signals in lymphocyte activation and disease S Feske
}

\author{
Address: NYU School of Medicine, Department of Pathology, New York, USA \\ from I2th Joint Meeting of the Signal Transduction Society (STS). Signal Transduction: Receptors, Mediators and Genes \\ Weimar, Germany. 29-3I October 2008 \\ Published: 26 February 2009 \\ Cell Communication and Signaling 2009, 7(Suppl I):A76 doi:10.1186/1478-8IIX-7-SI-A76
}

This abstract is available from: http://www.biosignaling.com/content/7/SI/A76

(c) 2009 Feske; licensee BioMed Central Ltd.

Calcium ions function as universal second messengers in virtually all eukaryotic cells including cells of the immune system where they are crucial for the function of $\mathrm{T}$ and $\mathrm{B}$ cells, mast cells and dendritic cells. The predominant mechanism regulating intracellular $\mathrm{Ca}^{2+}$ levels in cells of the adaptive immune system is store-operated $\mathrm{Ca}^{2+}$ influx through so-called $\mathrm{Ca}^{2+}$-release activated $\mathrm{Ca}^{2+}$ (CRAC) channels. We identified ORAI1 (also named CRACM1) as a pore subunit of the CRAC channel essential for the function of T cells and mast cells. ORAI1/CRAC channels are activated when intracellular $\mathrm{Ca}^{2+}$ stores are depleted. The resulting decrease in the $\mathrm{ER} \mathrm{Ca}^{2+}$ concentration is sensed by stromal interaction molecule 1 (STIM1) that is required for activation of ORAI1/CRAC channels. We showed that murine T cells lacking STIM1 exhibit severely impaired store-operated $\mathrm{Ca}^{2+}$ influx. T cells from mice lacking STIM1 or its paralogue STIM2 both showed significantly reduced cytokine production in vitro and a defect in regulatory $\mathrm{T}$ cell development as well as lympho- and myeloproliferation in vivo. Mutation of ORAI1 in humans is associated with severe combined immunodeficiency (SCID), increased susceptibility to infections and a failure to thrive. A similar defect is found in mice transgenic for the equivalent R93W mutation in murine ORAI1, which all but abrogates CRAC channel function and T cell activation. Taken together STIM1, STIM2 and ORAI1 are essential regulators of store-operated $\mathrm{Ca}^{2+}$ entry in cells of the immune system and other tissues. 